\title{
Sequentially triggered star formation in OB associations
}

\author{
Thomas Preibisch ${ }^{1}$ and Hans Zinnecker ${ }^{2}$ \\ ${ }^{1}$ Max-Planck-Institut für Radioastronomie, Auf dem Hügel 69, D-53121 Bonn, Germany \\ email: preib@mpifr-bonn.mpg.de \\ ${ }^{2}$ Astrophysikalisches Institut Potsdam, An der Sternwarte 16, D-14482 Potsdam, Germany \\ email: hzinnecker@aip.de
}

\begin{abstract}
We discuss observational evidence for sequential and triggered star formation in OB associations. We first review the star formation process in the Scorpius-Centaurus OB association, the nearest OB association to the Sun, where several recent extensive studies have allowed us to reconstruct the star formation history in a rather detailed way. We then compare the observational results with those obtained for other OB associations and with recent models of rapid cloud and star formation in the turbulent interstellar medium. We conclude that the formation of whole OB subgroups (each consisting of several thousand stars) requires large-scale triggering mechanisms such as shocks from expanding wind and supernova driven superbubbles surrounding older subgroups. Other triggering mechanisms, like radiatively driven implosion of globules, also operate, but seem to be secondary processes, forming only small stellar groups rather than whole OB subgroups with thousands of stars.
\end{abstract}

Keywords. stars: formation, open clusters and associations: individual (Sco OB2), shock waves, supernovae: general, ISM: clouds, bubbles, evolution

\section{Star formation in OB associations}

OB associations (Blaauw 1964) are loose, co-moving stellar groups containing Oand/or early B-type stars. As these associations are unstable against galactic tidal forces, they must be young $(\lesssim 30 \mathrm{Myr})$ entities, with most of their low-mass members (Briceno et al. 2006) still in their pre-main sequence (PMS) phase. OB associations are ideal targets for a detailed investigation of the initial mass function (IMF) and the star formation history, since they allow us to study the outcome of a recently completed star formation process. As noted by Blaauw (1964), many OB associations consist of distinct subgroups with different ages, which often seem to progress in a systematic way, suggesting a sequential, perhaps triggered formation scenario.

The massive stars in $\mathrm{OB}$ associations affect their environment by ionizing radiation, stellar winds, and, finally, supernova explosions. In their immediate neighborhood, these effects are mostly destructive, since they tend to disrupt the parental molecular cloud and thus terminate the star formation process (e.g. Herbig 1962). A little further away, however, massive stars may also stimulate new star formation. For example, the ionization front from a massive star can sweep up surrounding cloud material into a dense shell, which then fragments and forms new stars (the "collect and collapse" model; see Elmegreen \& Lada 1977; Zavagno et al. 2006). Also, shock waves from expanding windand/or supernova-driven superbubbles can trigger cloud collapse and star formation.

A general problem of scenarios of triggered star formation is that a clear proof of causality is hard to obtain. One can often see Young Stellar Objects (YSOs) and ongoing star formation near shocks caused by massive stars, suggesting triggered star formation. However, such morphological evidence alone does not constitute unequivocal proof for a 
triggered star formation scenario. It is not clear whether the shock really compressed an empty cloud and triggered the birth of the YSOs, or whether the YSOs formed before the shock wave arrived and the shock appears at the edge of an embedded star formation site simply because the associated dense cloud material has slowed it down. More insight can be gained if one can determine the ages of the YSOs and compare them to the moment in time at which the shock arrived. Agreement of these timings provides much more solid evidence for the triggered star formation scenario than the spatial alignment alone.

\section{Theoretical models for the formation of OB subgroups}

The classical model for the sequential formation of OB subgroups was developed by Elmegreen \& Lada (1977; see also Lada 1987). Low-mass stars are assumed to form spontaneously throughout the molecular cloud. As soon as the first massive stars form, their ionizing radiation and winds disperse the cloud in their immediate surroundings, thereby terminating the local star formation process. The OB star radiation and winds also drive shocks into other parts of the cloud. A new generation of massive stars is then formed in the dense shocked layers, and the whole process is repeated until the wave of propagating star formation reaches the edge of the cloud. According to this model, one would expect that (1) the low mass stars should be systematically older than the associated OB stars and should show a large age spread, corresponding to the total lifetime of the cloud, and that (2) the youngest OB subgroups should have the largest fraction of low-mass stars (as in these regions low-mass star formation continued for the longest period of time). Another model is based on the mechanism of radiation-driven implosion (e.g., Kessel-Deynet \& Burkert 2003). As an OB star drives an ionization shock front into the surrounding cloud, cores within the cloud are triggered into collapse by the shock wave. This model predicts that (1) the low-mass stars should be younger than the OB stars (which initiate their formation), and (2) one may expect to see an age gradient in the low-mass population (objects closer to the OB stars were triggered first and thus should be older than those further away; see, e.g. Chen, these proceedings).

The third model we consider here assumes that a shock wave driven by stellar winds and/or supernova explosions runs though a molecular cloud. Several numerical studies (e.g. Vanhala \& Cameron 1998) have found that the effect of the passing shock wave mainly depends on the type of the shock and its velocity: close to a supernova, the shock wave will destroy ambient clouds, but at larger distances, when the shock velocities have decreased to below $\sim 50 \mathrm{~km} \mathrm{~s}^{-1}$, cloud collapse can be triggered in the right circumstances. The distance from the shock source at which the shock properties are suitable for triggering cloud collapse depend on the details of the processes creating the shock wave (see Oey \& Garcia-Segura 2004), the structure of the surrounding medium, and the evolutionary state of the pre-impact core, but should typically range between $\sim 20$ pc and 100 pc. This model predicts that (1) low- and high-mass stars in the triggered subgroup should have the same age, and (2) the age spread in the new subgroup is small (since the triggering shock wave crossed the cloud quite quickly).

These quite distinct predictions of the different models can be compared to the observed properties (i.e. the IMF and the star formation history) of OB associations. The obvious first step of such a study is to identify the complete or a representative sample of the full stellar population of the association. While, at least in the nearby OB associations, the population of high- and intermediate mass stars has been revealed by Hipparcos, the low-mass members (which are usually too faint for proper-motion studies) are quite hard to find. Unlike stellar clusters, which can be easily recognized on the sky, OB associations are generally not so conspicuous because they extend over huge areas in the sky (often several hundred square-degrees for the nearest examples) and most of the faint stars in 
the area actually are unrelated fore- or background stars. Finding the faint low-mass association members among these field stars is often like finding needles in a haystack. However, the availability of powerful multiple-object spectrographs has now made large spectroscopic surveys for low-mass members possible. The young association members can, e.g., be identified by the strength of their $6708 \AA$ Lithium line, which is a reliable signature for young stars. Studies of the complete stellar population of OB associations are now feasible and allow us to investigate in detail the spatial and temporal relationships between high- and low-mass members.

\section{Triggered star formation in the Scorpius-Centaurus OB association}

At a distance of only $\sim 140 \mathrm{pc}$, the Scorpius-Centaurus (ScoCen) association is the OB association nearest to the Sun. It contains at least $\sim 150$ B stars which concentrate in the three subgroups Upper Scorpius (USco), Upper Centaurus-Lupus (UCL), and Lower Centaurus-Crux (LCC). The ages for the B-type stars in the different subgroups, derived from the main sequence turnoff in the HR diagram, were found to be $\sim 5 \mathrm{Myr}$ for USco, $\sim 17$ Myr for UCL, and 16 Myr for LCC (de Geus et al. 1989; Mamajek et al. 2002).

Upper Scorpius is the best studied part of the ScoCen complex. de Zeeuw et al. (1999) identified 120 stars listed in the Hipparcos Catalogue as genuine members of high- and intermediate mass $\left(\sim 20-1.5 M_{\odot}\right)$. After the first systematic large-scale search for lowmass members of USco by Walter et al. (1994), we have performed extensive spectroscopic surveys for further low-mass members with wide-field multi-object spectrographs at the Anglo-Australian Observatory. These observations are described in detail in Preibisch et al. (1998) and Preibisch et al. (2002, P02 hereafter), and ultimately yielded a sample of 250 low-mass members in the mass range $\sim 0.1 M_{\odot}$ to $\sim 2 M_{\odot}$. In combination with the Hipparcos sample of high- and intermediated mass stars, this large sample allowed P02 to study the properties of the full stellar population in USco on the basis of a statistically robust and well defined sample of members. The main results of our detailed analysis of this sample can be summarized as follows: (1) The stellar mass function in USco is consistent with recent field star and cluster IMF determinations. (2) Highas well as low-mass stars have a common mean age of 5 Myr. (3) The spread seen in the HRD, that may seem to suggest an age spread, can be fully explained by the effects of the spread of individual stellar distances, unresolved binary companions, and the photometric variability of the young stars. The observed HRD provides no evidence for an age dispersion, although small age spreads of $\sim 1-2$ Myr cannot be excluded. (4) The initial size of the association and the observed internal velocity dispersion of the members yield a stellar crossing time of $\sim 20 \mathrm{Myr}$.

A very important implication of these results is that the observed age spread of at most $\leqslant 1-2 \mathrm{Myr}$ is much smaller than the stellar crossing time of $\sim 20 \mathrm{Myr}$. This clearly shows that some external agent must have coordinated the onset of the star formation process over the full spatial extent of the association. In fact, a very suitable trigger is actually available: The structure and kinematics of the large H I loops surrounding the ScoCen association suggest that a shock wave from the older UCL group, driven by stellar winds and supernova explosions, passed through the USco region just about 5 Myr ago (de Geus 1992), which agrees very well with the ages of the USco members. A scenario for the star formation history of USco consistent with the observational results described above is shown in Fig. 1. The shock-wave from UCL initiated the formation of some 2500 stars in USco, including 10 massive stars upwards of $10 M_{\odot}$. The newborn massive stars immediately started to destroy the cloud from inside by their ionizing radiation and their strong winds and terminated the star formation process; this explains 


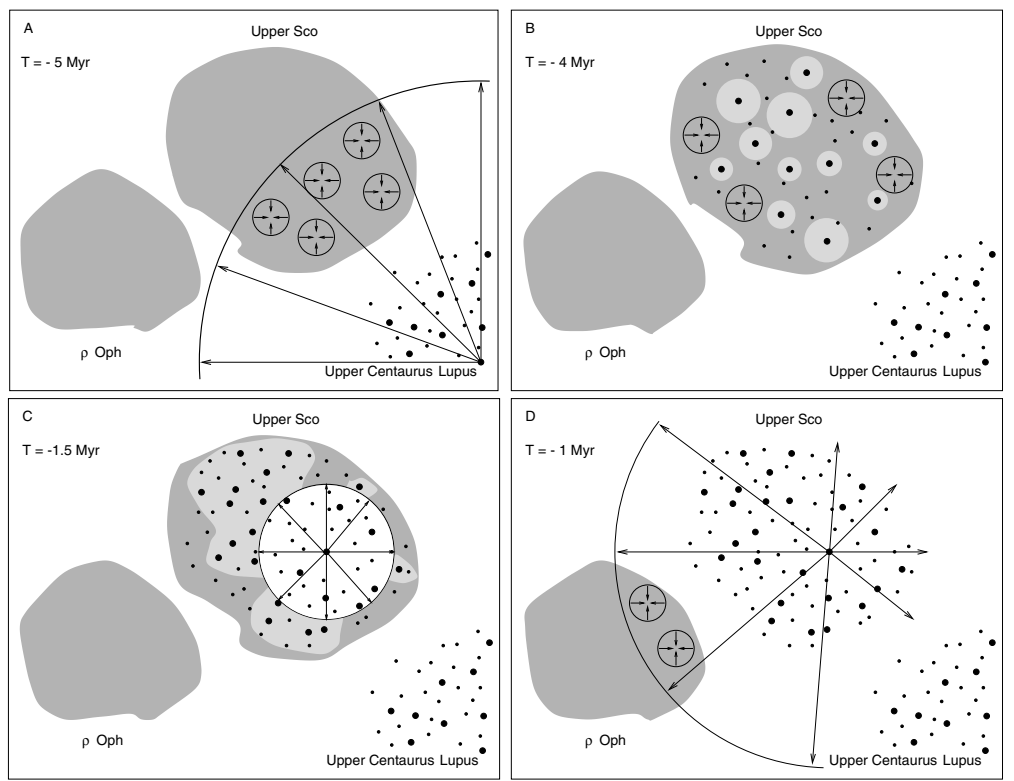

Figure 1. Schematic view of the star formation history in the Scorpius-Centaurus association (from Preibisch \& Zinnecker 1999). Molecular clouds are shown as dark regions, high-mass and low-mass stars as large resp. small dots. For further details on the sequence of events see text.

the narrow age distribution. About 1.5 Myr ago, the most massive star in USco exploded as a supernova and created another strong shock wave, which fully dispersed the USco molecular cloud. This shock wave must have crossed the adjacent $\rho$ Oph molecular cloud within the last 1 Myr (de Geus 1992), and thus seems to have triggered the strong star formation activity we witness right now in the L 1688 cloud (see Motte, André, \& Neri 1998). Yet another region in which the shock from USco seems to have triggered star formation is the Lupus 1 cloud (see, e.g., Tachihara 2001).

While this scenario provides a good explanation of the star formation history, a potential problem is its implicit assumption that the USco and $\rho$ Oph molecular clouds existed for many Myr without forming stars before the triggering shock waves arrived (otherwise one should see large age spreads in the stellar populations). Elmegreen (2000), Hartmann et al. (2001), and other authors provided several convincing arguments that the lifetime of molecular clouds is much shorter than previously thought, and the whole process of molecular cloud formation, star formation, and cloud dispersal (by the feedback of the newly formed stars) occurs on timescales of a few $(\lesssim 5)$ Myr. It is now thought that molecular clouds form by the interaction of turbulent convergent flows in the interstellar medium that accumulate matter in some regions. Once the $\mathrm{H}_{2}$ column density becomes high enough to provide effective self-shielding against the ambient UV radiation field, conversion of atomic $\mathrm{H}$ into molecular $\mathrm{H}_{2}$ quickly follows (e.g., Glover \& Mac Low 2006), and star formation may start soon afterwards (e.g., Clark et al. 2005).

This new paradigm for the formation and lifetime of molecular clouds may seem to invalidate the idea of a shock wave hitting a pre-existing molecular cloud and triggering star formation. Nevertheless, the basic scenario for the sequence of processes in ScoCen may still be valid. As pointed out by Hartmann et al. (2001), wind and supernova shock waves from massive stars are an important kind of driver for ISM flows, and are especially well suited to create coherent large-scale flows. Only large-scale flows are able to form large molecular clouds, in which whole OB associations can be born. An updated scenario 
for ScoCen could be as follows: Initially, the winds of the OB stars created an expanding superbubble around UCL which interacted with flows in the ambient ISM and swept up clouds in some places. When supernovae started to explode in UCL (note that there were presumably some 6 supernova explosions in UCL up to the present day), these added energy and momentum to the wind-blown superbubble and accelerated its expansion. The accelerated shock wave (now with $v \sim 20-30 \mathrm{~km} \mathrm{~s}^{-1}$ ) crossed a swept-up cloud in the USco area, and the increased pressure due to this shock triggered star formation.

This scenario does not only explain the temporal sequence of events in a way consistent with the ages of the stars and the kinematic properties of the observed HI shells. The following points provide further evidence: (1) The model (see Fig. 3 in Hartmann et al. 2001) predicts that stellar groups triggered in swept-up clouds should be moving away from the trigger source. A look at the centroid space motions of the ScoCen subgroups (de Bruijne 1999) actually shows that USco is moving away from UCL with a velocity of $\sim 5( \pm 3) \mathrm{km} \mathrm{s}^{-1}$. Furthermore, (2) a study by Mamajek \& Feigelson (2001) revealed that several young stellar groups, including the $\eta$ Cha cluster, the TW Hydra association, and the young stars associated with the CrA cloud, move away from UCL at velocities of about $10 \mathrm{~km} \mathrm{~s}^{-1}$; tracing their current motions back in time shows that these groups were located near the edge of UCL 12 Myr ago (when the supernova exploded). Finally, (3) the model also predicts that molecular clouds are most efficiently created at the intersection of two expanding bubbles. The Lupus I cloud, which is located just between USco and UCL, may be a good example of this process. Its elongated shape is very consistent with the idea that it was swept up by the interaction of the expanding superbubble from USco and the (post-SN) bubble created by the winds of the remaining early B stars in UCL.

\section{Triggered star formation in other OB associations}

While model and observations agree quite well for ScoCen, it is important to consider how general these findings are. We first note that numerous other regions show similar patterns of sequential star formation, e.g. the superbubbles in W3/W4a (Oey et al. 2005), or the supergiant shell region in IC 2574 (Cannon et al. 2005). Some OB associations, however, show subgroups with similar ages, which cannot have formed sequentially. This could nevertheless fit into the proposed scenario, if we consider the example of Hen 206 in the LMC (Gorjian 2004), where a supernova driven shock wave from a $\sim 10$ Myr old OB association has created a huge expanding superbubble, at the southern edge of which stars are forming in a giant swept-up molecular cloud. This cloud contains several discrete peaks, in each of which an entire new OB subgroup seems to be forming. This could be an example of the simultaneous formation of several OB subgroups.

A second important aspect is that most nearby OB associations seem to share some key properties: (1) Their mass function is consistent with the field IMF, without much evidence for any IMF variations. (2) High- and low-mass stars generally have the same ages and thus have formed together, not one first and the other later. (3) In most regions, age spreads are remarkably small, often much smaller than the stellar crossing time. These properties are more consistent with the model of large-scale, fast triggering by passing shock waves from wind and supernova driven expanding (super-)bubbles than with the other triggering mechanisms mentioned above.

Nevertheless, other triggering mechanisms seem to operate simultaneously, at least in some regions. For example, the IC 1396 H II region in the Cep OB2 association contains several globules, which are strongly irradiated by the central O6 star. Spitzer observations of the globule VDB 142 (Reach et al. 2004) and Chandra X-ray observations of another globule (Getman et al. 2006) have revealed several very young stellar objects within these 
globules, all of which are located close to the illuminated edge of the globules, providing evidence for triggered star formation. The triggering mechanism at work here (radiationdriven implosion of globules) is obviously different from the large-scale triggering by expanding wind/supernova driven superbubbles. However, just a handful of stars are formed in these globules and thus this mechanism seems to be a small-scale, secondary process, which does not seem capable of forming whole OB subgroups.

\section{Summary and conclusions}

OB associations with subgroups showing well defined age sequences and small internal age spreads suggest a large-scale triggered formation scenario, presumably due to supernova/wind driven shock waves. Expanding, initially wind driven superbubbles around OB groups produce coherent large-scale ISM flows that form new clouds; supernova shock waves then compress these newly formed clouds and trigger the formation of whole OB subgroups (several thousand stars) in locations with suitable conditions. Other triggering mechanisms (e.g. radiatively driven implosion) may operate simultaneously, but seem to form only small groups of stars and thus appear to be secondary processes.

\section{References}

Blaauw, A. 1964, ARAA 2, 213

Briceno, C., Preibisch, Th., Sherry, W., Mamajek, E., Mathieu, R., Walter, F. \& Zinnecker, H. 2006, in: B. Reipurth, D. Jewitt, \& K. Keil (eds.), Protostars 8 Planets V (Tucson: Univ. of Arizona), in press [astro-ph/0602446]

Cannon, J. M., et al. 2005, ApJ 630, L37

Clark, P. C., Bonnell, I. A., Zinnecker, H. \& Bate, M. R. 2005, MNRAS 359, 809

de Bruijne, J. H. J. 1999, MNRAS 310, 585

de Geus, E. J. 1992, A\& A 262, 258

de Geus, E. J., de Zeeuw, P. T. \& Lub, J. 1989, A\&A 216, 44

Elmegreen, B. G. 2000, ApJ 530, 277

Elmegreen, B. G. \& Lada, C. J. 1977, ApJ 214, 725

Getman, K. V., Feigelson, E. D., Garmire, G., et al. 2006, ApJ in press [astro-ph/0607006]

Glover, S. C. O. \& Mac Low, M.-M. 2006, ApJ in press [astro-ph/0605121]

Gorjian, V., et al. 2004, ApJS 154, 275

Hartmann, L., Ballesteros-Paredes, J. \& Bergin, E. A. 2001, ApJ 562, 852

Herbig, G. H. 1962, ApJ 135, 736

Kessel-Deynet, O. \& Burkert, A. 2003, MNRAS 338, 545

Lada, C. J. 1987, IAU Symp. 115: Star Forming Regions, 115, 1

Mamajek, E. E., \& Feigelson, E. D. 2001, in: R. Jayawardhana \& T. Greene (eds.), Young Stars Near Earth: Progress and Prospects (ASP-CS), 244, 104

Mamajek, E. E., Meyer, M. R. \& Liebert, J. 2002, AJ 124, 1670

Motte, F., André, P. \& Neri, R. 1998, A\&\&A 336, 150

Oey, M. S. \& Garcia-Segura, G. 2004, ApJ 613, 302

Oey, M. S., Watson, A. M., Kern, K. \& Walth, G. L. 2005, AJ 129, 393

Preibisch, Th. \& Zinnecker, H. 1999, AJ 117, 2381

Preibisch, Th., Guenther, E., Zinnecker, H., et al. 1998, A\& A 333, 619

Preibisch, Th., Brown, A. G. A., Bridges, T., Guenther, E. \& Zinnecker, H. 2002, AJ 124, 404

Reach, W. T., et al. 2004, ApJS 154, 385

Tachihara, K., Toyoda, S., Onishi, T., et al. 2001, PASJ 53, 1081

Vanhala, H. A. T \& Cameron, A. G. W. 1998, ApJ 508, 291

Walter, F. M., Vrba, F. J., Mathieu, R. D., Brown, A. \& Myers, P. C. 1994, AJ 107, 692

Zavagno, A., Deharveng, L., Comerón, et al. 2006, A\&\&A 446, 171

de Zeeuw, P. T., Hoogerwerf, R., de Bruijne, J. H. J., et al. 1999, AJ 117, 354 


\section{Discussion}

KRUMHOLz: If triggering by OB associations is a dominant mechanism for star formation, why are there spiral arms? Creating clouds by sweeping up gas would not produce a spiral pattern.

PREIBISCH: Our argument for the importance of large-scale triggering by expanding wind- and supernova-driven shocks from $\mathrm{OB}$ associations does not imply that this mechanism alone dominates the whole galactic star formation; other mechanisms are at work simultaneously. Triggering by large-scale shocks may be efficient only in suitable locations and in the right circumstances. Spiral shocks increase the density of the interstellar material and create large-scale flows; in this way they may set the stage for wind and supernova shock triggering to work during spiral arm passages.

TAN: You argue that evidence for SN triggering of star formation is the small age spread compared to the crossing time. However, the relevant crossing time is that of the stars and gas at the embedded stage. It is hard to measure this in clusters that have been expanding for more than several Myr and expelled their natal gas. When we look at embedded clusters, like the ONC (Tan et al. 2006), we find age spreads that are greater than the crossing time. (The high stellar density and the background obscuration mean that line of sight contamination is not significant for the ONC).

PREIBISCH: We agree that the relevant crossing time is that of the initial configuration, i.e. at the time of star formation and not today. Our determination of the crossing time is based on the initial size of Upper Sco of $\sim 25 \mathrm{pc}$, which we derived from the spatial structure and kinematics of the association (see Preibisch et al. 2002 for details). Upper Sco was apparently not born as a dense cluster. With respect to the ONC, we would like to note that the presence of a large age spread (Tan et al. 2006) is not fully clear. Individual stellar ages derived from the HRD must not be taken too literally, because they are easily adulterated by effects such as the presence of unresolved binary companions, photometric variability, and errors in the luminosity determination. Thus, the dispersion of the ages determined from the HRD is always only an upper limit to the true age dispersion. We note that HST photometry of the inner ONC by Prosser et al. (1994), taking into account these effects, showed that "only a small fraction of the stars (if truly members) have been born outside of a 1 Myr era of star formation." Another factor is contamination: as the ONC coincides with the center of the $\sim 5$ Myr old Ori OB1c association, some of the apparently older ONC stars may in fact be Ori OB1c members. Furthermore, star formation does not continue in the ONC but in the molecular cloud behind the ONC. In view of these arguments, it does not seem obvious that the age spread in the $\mathrm{ONC}$ is greater than the crossing time.

BreitschwerdT: Some of the stars that belong to UCL and LCC are members of a moving group that was born about $20 \mathrm{Myr}$ ago and its high mass members generated the Local Bubble before entering the Loop I region just 1-3 Myr ago. How would explosions from these stars affect your model of triggering star formation by a cluster with a single age?

PREIBISCH: The suggested star formation history would not be affected by SN explosions during the last $5 \mathrm{Myr}$ (when Upper Sco was formed). 
BONNELL: How can you relate your small age spreads to the large age spreads reported for the ONC?

PREIBISCH: The large age spreads that are apparently seen in some regions may in fact, at least in some cases, be caused by projection effects, i.e., we see several stellar groups with different ages projected onto each other. A good example may be the ONC region, with the $\sim 10^{5}$ yr old $\mathrm{BN}$ region, in the background of the $1 \mathrm{Myr}$ Trapezium cluster, which is seen in projection behind the Ori OB1c association (several My old).

CHU: IAU commission 34 (Div VI) includes both ISM and star formation, but the communication between ISM and star formation researchers is lacking. "SN/wind shocks" and "bubbles" are used by star formation people loosely with different definitions from the ISM people. SNRs, when they are still recognizable as SNRs, are too violent to trigger star formation. SNRs and stellar winds collectively form "superbubbles," and superbubbles can trigger star formation. We really agree on all physical processes, but we need to use terminology consistently.

PREIBISCH: The expanding bubbles in Sco Cen are superbubbles, driven by several stars and SN explosions. I agree that SNR are too violent to trigger star formation, but at large distances from the $\mathrm{SN}(>20-30 \mathrm{pc})$ the shock wave may be able to trigger star formation. 